ARTIGOS

Submetido em 04.12.2019. Aprovado em 26.08.2020

Avaliado pelo processo de double-blind review. Editora Científica: Darcy Mitiko Mori Hanashiro

Versão traduzida | DOI: http://dx.doi.org/10.1590/So034-759020210405X

\title{
INFLUÊNCIA DO CAPITAL PSICOLÓGICO NA APRENDIZAGEM INTERNA EM EQUIPES: PAPEL MEDIADOR DA ESTRUTURA PERCEBIDA DA EQUIPE
}

\author{
The influence of psychological capital on internal learning in teams: the mediating role of the \\ perceived team structure

\section{La influencia del capital psicológico en el aprendizaje interno del equipo: El papel mediador de la estructura percibida del equipo}

Rosa Lutete Geremias' ${ }^{1}$ lutetegeremias@hotmail.com | ORCID: 0000-0002-7267-7294 Miguel Pereira Lopes ${ }^{1}$ | mplopes@iscsp.ulisboa.pt | ORCID: 0000-0002-7720-8657 André Escórcio Soares ${ }^{2,3}$ | andre.soares@coventry.ac.uk | ORCID: 0000-0001-7135-3530

${ }^{1}$ Universidade de Lisboa, Instituto Superior de Ciências Sociais e Políticas, Lisboa, Portugal

${ }^{2}$ Coventry University, Faculty of Health and Life Sciences, Coventry, Reino Unido

${ }^{3}$ Nicolaus Copernicus University, Faculty of Economic Sciences and Management, Toruń, Polânia

\section{RESUMO}

Pesquisas anteriores sobre a aprendizagem interna concentraram-se em avaliar como as taxas de aprendizagem observadas afetam as interações colaborativas entre os vários atores. Assim, agregamos valor a essa literatura ao analisar a aprendizagem interna em equipes e o papel do capital psicológico nesse processo. Este estudo tem como objetivo analisar o papel mediador da estrutura percebida da equipe na relação entre capital psicológico positivo e aprendizagem interna em equipes. Para tanto, um questionário foi aplicado a 480 estudantes universitários para testar essa relação, utilizando a metodologia de modelagem de equações estruturais. Os resultados confirmaram o papel mediador da estrutura percebida da equipe na relação entre capital psicológico e aprendizagem interna em equipes. Argumentamos que este trabalho reforça o papel do capital psicológico em contextos acadêmicos. Por outro lado, devido às rápidas mudanças verificadas atualmente na sociedade, os estudantes universitários, que são futuros funcionários, precisam desenvolver o capital psicológico, a fim de obter melhores resultados de aprendizagem.

PALAVRAS-CHAVE | Capital psicológico positivo, estrutura percebida da equipe, aprendizagem interna em equipes, processo de aprendizagem, estudantes de graduação.

\section{ABSTRACT}

Whereas past research on internal learning has focused on evaluating how the observed learning rates affect collaborative interactions among the various actors, we extend this literature by analyzing internal learning in teams and the role of psychological capital in this process. This study aims to analyze the mediating role of the perceived team structure in the relationship between positive psychological capital and internal learning in teams. Therefore, a self-report questionnaire was applied to 480 college students to test this relationship, using structural equation modeling. The results confirmed the mediating role of the perceived team structure in the relationship between psychological capital and internal learning in teams. Our work underlines the role of psychological capital in academic settings. On the other hand, due to rapid changes in today's society, university students who will be future employees need to cultivate psychological capital in order to achieve better learning outcomes.

KEYWORDS I Positive psychological capital, perceived team structure, internal learning in teams, learning process, undergraduate students.

\section{RESUMEN}

La investigación previa sobre el aprendizaje interno se ha centrado en evaluar cómo las tasas de aprendizaje observadas afectan las interacciones colaborativas entre los diversos actores. Por lo tanto, agregamos valor a esta literatura al analizar el aprendizaje interno en equipos y el papel del capital psicológico en este proceso. Este estudio tiene como objetivo analizar el papel mediador de la estructura percibida del equipo en la relación entre el capital psicológico positivo y el aprendizaje interno en los equipos. Por lo tanto, se aplicó un cuestionario a 480 estudiantes universitarios para probar esta relación, utilizando la metodología de modelado de ecuaciones estructurales. Los resultados confirmaron el papel mediador de la estructura percibida del equipo en la relación entre el capital psicológico y el aprendizaje interno en los equipos. Sostenemos que este trabajo refuerza el papel del capital psicológico en contextos académicos. Por otro lado, debido a los rápidos cambios observados en la sociedad actual, los estudiantes universitarios que son futuros empleados necesitan desarrollar capital psicológico para obtener mejores resultados de aprendizaje.

PALABRAS CLAVE / Capital psicológico, estructura de equipo percibida, aprendizaje interno del equipo, proceso de aprendizaje, estudiantes de pregrado. 


\section{INTRODUÇÃO}

Estudos sobre a aprendizagem individual demonstram que ela se encontra no cerne das mudanças e da renovação (Argote \& Miron-Spektor, 2011). Atividades colaborativas que levam à aprendizagem individual são consideradas críticas para alcançar diferentes resultados positivos (Song, Lim, Kang, \& Kim, 2014). A identificação do grau de envolvimento dos indivíduos no processo de aprendizagem tem sido considerada a principal questão em pesquisas anteriores (Bresman, 2010). Assim, as teorias de aprendizagem tendem a enfatizar a orientação para metas e as interações colaborativas entre os vários atores, com o intuito de minimizar erros e melhorar o desempenho individual (Bunderson \& Reagans, 2011).

0 estudo dos antecedentes da aprendizagem individual concentra-se essencialmente em explicar as diferenças nas taxas de aprendizagem observadas e avalia como outros fatores, como a estrutura percebida da equipe, interagem para influenciar a aprendizagem (Bresman \& Zellmer-Bruhn, 2013). Embora tais abordagens indiquem determinados processos de aprendizagem individual, existe, ainda, a necessidade de uma construção mais teórica e de aplicações práticas eficazes que analisem de modo mais abrangente como os fatores de aprendizagem são facilitados (Yoon \& Kayes, 2016). Conforme defendem Bunderson e Boumgarden (2010), saber mais sobre os fatores antecedentes que afetam a aprendizagem individual é uma contribuição valiosa, pois melhora nossa compreensão dos processos de aprendizagem.

O capital psicológico positivo (PsyCap) é um construto de ordem superior que integra os recursos psicológicos (por exemplo, autoeficácia), recursos motivacionais (por exemplo, esperança) e outros recursos psicológicos positivos (por exemplo, otimismo e resiliência). Argumentamos que o PsyCap pode ser um dos antecedentes mencionados acima, pois é considerado um preditor útil de resultados importantes em contextos acadêmicos (Datu \& Valdez, 2019; Luthans, Luthans, \& Jensen, 2012). Para Siu, Bakker e Jiang (2014), estudantes e funcionários trabaIham para atingir metas específicas, como bom desempenho acadêmico, conclusão do curso e obtenção de um diploma acadêmico. Eles também argumentaram que o PsyCap é um recurso pessoal útil que auxilia o atingimento de objetivos, podendo, portanto, ajudar estudantes universitários a enfrentarem os desafios de seus estudos.

Pesquisas anteriores indicam a necessidade de mais estudos sobre como integrar o capital psicológico no processo de aprendizagem (Daspit, Mims, \& Zavattaro, 2015). Herrmann (2013) observa que "existem poucos estudos voltados ao exame da aprendizagem interna para além do contexto organizacional". O presente estudo pretende preencher essa lacuna nas pesquisas, na medida em que descreve uma abordagem da incorporação do capital psicológico e da aprendizagem interna em equipes. 0 objetivo deste estudo é, portanto, analisar o papel mediador da estrutura percebida da equipe na relação entre PsyCap e aprendizagem interna em equipes.

Daspit et al. (2015) apresentaram evidências empíricas para sustentar a influência positiva do capital psicológico no processo de aprendizagem de estudantes durante um curso on-line. Entretanto, nenhuma evidência foi coletada a partir da análise do papel mediador da estrutura percebida da equipe na relação entre o capital psicológico e outras variáveis, como a aprendizagem interna, o que acreditamos ser uma importante contribuição teórica deste estudo.

Para Schaubroeck, Carmeli, Bhatia e Paz (2016), se não houver um processo de aprendizagem adequado, os membros da equipe terão dificuldade em desenvolver e implementar melhores práticas para coordenar suas ações quando mudanças ambientais exigirem novas abordagens. Argumentamos que este estudo pode levar a certas implicações práticas, pois a aprendizagem interna em equipes permite que os estudantes se concentrem na compreensão de conceitos para relacionarem novas ideias a conhecimentos e experiências anteriores. 
O restante deste trabalho está organizado da seguinte forma: primeiramente, apresentamos uma revisão da literatura e a justificativa das hipóteses; em seguida, definimos a opção e os procedimentos metodológicos; depois, apresentamos uma análise dos resultados; finalmente, discutimos os resultados e concluímos com as implicações de nossos achados.

\section{REVISÃO DA LITERATURA E HIPÓTESES}

\section{Capital psicológico}

Estudos teóricos e empíricos têm apoiado a tese de que o PsyCap é um construto emergente fundamental relacionado a diferentes resultados positivos, favorecendo uma avaliação positiva das circunstâncias e da probabilidade de sucesso, tendo o esforço e a perseverança como fatores de motivação (Heled, Somech, \& Waters, 2015; Luthans, Avey, Avolio, \& Peterson, 2010).

De acordo com Luthans, Avey, Avolio, Norman e Combs (2006), o PsyCap é definido, de maneira ampla, como o estado psicológico positivo de desenvolvimento de um indivíduo que se caracteriza por: (1) possuir confiança para assumir tarefas desafiadoras e realizar os esforços necessários para obter êxito (autoeficácia); (2) adotar uma abordagem positiva em relação ao sucesso, tanto no presente quanto no futuro (otimismo); (3) perseverar no atingimento dos objetivos e, quando necessário, redirecionar os caminhos de acordo com os objetivos para alcançar êxito (esperança); e (4) ser capaz de superar obstáculos e adversidades para alcançar êxito (resiliência), sem nunca desistir. Em geral, as pesquisas teóricas consideram quatro capacidades de PsyCap, a saber: autoeficácia, otimismo, esperança e resiliência (Newman, Ucbasaran, Zhu, \& Hirst, 2014). Essas capacidades estão inter-relacionadas, assim, se uma delas for afetada (por exemplo, o otimismo), outras (por exemplo, a esperança, a autoeficácia e/ou a resiliência) provavelmente também serão afetadas ao longo do tempo (Peterson, Luthans, Avolio, Walumbwa, \& Zhang, 2011).

Os atributos dessas capacidades podem ser definidos da seguinte forma: (1) autoeficácia - refere-se à confiança individual, que eleva os níveis de motivação, e aos recursos cognitivos, ambos os quais resultam em um desempenho excelente (Bandura, 1982; Newman et al., 2014). (2); otimismo - um aumento dos atributos e expectativas positivas em relação à realização de eventos futuros (Harms \& Luthans, 2012); (3) esperança - um conjunto cognitivo derivado da interação entre agência (determinação orientada para atingir metas) e planos direcionais (etapas de planejamento que levam ao atingimento dos objetivos) (Walumbwa, Luthans, Avey, \& Oke, 2011); e (4) resiliência - capacidade que permite aos indivíduos enfrentar a adversidade, ou se ajustar a ela de maneira positiva (King, Newman, \& Luthans, 2015).

Chen e Lim (2012) apontaram que essas quatro capacidades psicológicas positivas básicas tendem a afetar os resultados de maneira sinérgica, e são melhor representadas por um construto de ordem superior denominado PsyCap. Avey (2014) argumenta que o PsyCap não é uma dimensão única (por exemplo, simplesmente otimismo), mas sim uma variação compartilhada de todas as quatro dimensões. Essas quatro capacidades psicológicas positivas, portanto, são parte de um construto maior de segunda ordem, denominado PsyCap.

A combinação das quatro capacidades psicológicas (autoeficácia, otimismo, esperança e resiliência) proporciona um alto nível de capital psicológico que permite ao indivíduo concentrar-se na execução de tarefas e na busca do êxito na conclusão dessas tarefas (Peterson et al., 2011). Mais especificamente, tais capacidades 
ARTIGOS | INFLUÊNCIA DO CAPITAL PSICOLÓGICO NA APRENDIZAGEM INTERNA EM EQUIPES: PAPEL MEDIADOR DA ESTRUTURA PERCEBIDA DA EQUIPE

Rosa Lutete Geremias | Miguel Pereira Lopes1 | André Escórcio Soares

foram determinadas para atender aos critérios de inclusão, pesquisa e validação das medidas de capital psicológico como uma teoria, permitindo, assim, que esta seja amplamente desenvolvida e tenha maior impacto (Walumbwa et al., 2011).

De acordo com Daspit et al. (2015), o capital psicológico é um conjunto de motivações individuais que estimulam o desenvolvimento de resultados de aprendizagem. A relação esperada entre capital psicológico e aprendizagem individual, entretanto, não é óbvia e permanece aberta a investigações futuras.

\section{Aprendizagem interna em equipes}

A capacidade de aprendizagem e a adaptação individual dos atores são fundamentais para o desempenho e o sucesso de longo prazo das organizações (Argote \& Miron-Spektor, 2011).

Edmondson (1999, p. 353) conceitualizou a aprendizagem individual em equipes como um processo contínuo de reflexão e ação, caracterizado por fazer perguntas, buscar feedback, experimentar, refletir sobre os resultados e discutir erros ou resultados inesperados de ações.

Esse processo permite uma compreensão mais abrangente e robusta das possibilidades futuras, por meio do uso de diferentes informações derivadas das percepções e perspectivas dos membros da equipe, e pela análise crítica e avaliação de ações passadas (Bunderson \& Reagans, 2011).

0 trabalho individual em uma equipe permite a comunicação e o compartilhamento de conhecimentos transformando, assim, as perspectivas individuais percebidas dos membros da equipe em conceitos explícitos que podem influenciar positivamente outros membros (Kayes, Kayes, \& Kolb, 2005; Kostopoulos, Spanos, \& Prastacos, 2013). As diferentes perspectivas e experiências de cada indivíduo são também aquilo que possibilita 0 processo de aprendizagem interpessoal por meio do compartilhamento formal ou informal de conhecimentos (Bunderson \& Reagans, 2011).

Portanto, a aprendizagem interna baseia-se nas próprias experiências dos membros da equipe na geração de novas soluções e na adoção de novas abordagens por meio de interações entre os membros (Bresman \& Zellmer-Bruhn, 2013). A aprendizagem interna pode proporcionar à equipe oportunidades de aprender sobre todos os aspectos de seu trabalho (Bresman, 2010). De acordo com Bresman e Zellmer-Bruhn (2013), o processo de aprendizagem interna é crucial porque os membros da equipe podem obter e dominar as informações mais recentes sobre tecnologias e mercados (aprendizagem externa); porém, sem um processo eficaz de aprendizagem interna, tais conhecimentos podem não ser adequadamente aproveitados (Bresman \& Zellmer-Bruhn, 2013).

Nesse contexto, uma análise da aprendizagem individual em equipe deve considerar essencialmente a aprendizagem interna dos membros da equipe, uma vez que fatores que promovem a aprendizagem interna acabam impulsionando a aprendizagem externa (Bresman \& Zellmer-Bruhn, 2013). Isso é particularmente importante diante do fato de que a aprendizagem individual pode ser aprimorada pela inclusão de tarefas novas e desafiadoras (Jiang, Jackson, \& Colakoglu, 2015).

Tem sido sugerido que o processo de aprendizagem é o principal mecanismo psicológico e comportamental pelo qual os indivíduos adquirem as habilidades e conhecimentos necessários para enfrentar e ter sucesso em ambientes competitivos (Yoon \& Kayes, 2016). Apesar da conceitualização teórica e prática do PsyCap e do processo de aprendizagem, poucos são os estudos que analisam a influência do capital psicológico no processo de aprendizagem individual. Este estudo, portanto, integra esses dois conceitos. 


\section{O capital psicológico e a aprendizagem interna em equipes}

A influência do PsyCap no processo de aprendizagem é apontada como relevante por autores como Yoon e Kayes (2016). Esses autores enfatizam que, em muitas situações, o processo de aprendizagem exige que os funcionários enfrentem desafios, reflitam sobre os erros e realizem com sucesso tarefas que envolvem risco.

Quando os membros de uma equipe estão ativamente envolvidos em discussões que visam a atingir os objetivos declarados, eles têm a oportunidade de trocar ideias, compartilhar percepções sobre as diferentes alternativas identificadas e, consequentemente, multiplicar o atingimento de seus objetivos, aumentando, assim, suas capacidades psicológicas positivas (Dawkins, Martin, Scott, \& Sanderson, 2013).

Segundo Huang e Luthans (2014), nesse contexto, indivíduos com alto nível de capital psicológico tendem a se diferenciar dos demais pela capacidade de enfrentar tarefas desafiadoras, o que pode impulsionar o processo de aprendizagem. Assim, o processo de aprendizagem interna requer reflexão colaborativa sobre as experiências da equipe, com o objetivo de melhorar a capacidade de colaboração e os padrões de interação de seus membros (Schaubroeck et al., 2016). É nesse sentido que propomos as seguintes hipóteses:

H1: O capital psicológico influencia positivamente a aprendizagem interna em equipes.

\section{O capital psicológico e a estrutura percebida da equipe}

Segundo Bunderson e Boumgarden (2010), a estrutura percebida da equipe refere-se à divisão do trabalho em tarefas e às relações que se estabelecem de acordo com as tarefas atribuídas. Bresman e Zellmer-Bruhn (2013) apontam duas dimensões principais na estrutura percebida da equipe: (1) especialização, isto é, a divisão horizontal do trabalho (por exemplo, tarefas e papéis); e (2) formalização, isto é, a articulação explícita de objetivos, prioridades e procedimentos.

Pesquisas anteriores têm destacado os benefícios da estrutura percebida da equipe no que diz respeito a questões de eficiência, previsibilidade e cumprimento das tarefas. Por exemplo, Bresman e Zellmer-Bruhn (2013) enfatizam que as equipes podem criar estruturas para si mesmas que atendam às demandas de suas tarefas individuais. Também foi demonstrado que a estrutura percebida da equipe pode ser considerada quando há pouco planejamento e poucos procedimentos especificando como o trabalho deve ser realizado (Bunderson \& Boumgarden, 2010).

Indivíduos com alto nível de capital psicológico tendem a gerar expectativas positivas que os estimulam a alcançar objetivos definidos e lidar com situações adversas (Newman et al., 2014). Por outro lado, outros autores, como Bresman e Zellmer-Bruhn (2013), destacaram a relevância da estrutura percebida da equipe, argumentando que essa estrutura permite que as delimitações da equipe sejam definidas pela divisão de tarefas e alocação de responsabilidades por subtarefa.

A divisão do trabalho possibilita a execução de tarefas específicas que os membros da equipe devem realizar para atingir os objetivos propostos, o que requer altos níveis de capital psicológico para um melhor desempenho (Salas, Shuffler, Thayer, Bedwell, \& Lazzara, 2014). Como afirmam Siu et al. (2014), indivíduos com altos níveis de PsyCap promovem uma abordagem positiva dentro de uma equipe, e podem efetivamente melhorar os processos, prioridades e procedimentos de trabalho de estudantes universitários.

Ortega-Maldonado e Salanova (2017) constataram que estudantes utilizam suas capacidades psicológicas para definir tarefas ou atingir objetivos acadêmicos. Essa pesquisa também mostra que, em inúmeras situações 
acadêmicas desafiadoras, os estudantes podem necessitar de um alto nível de capital psicológico para realizar o esforço necessário para completar as tarefas definidas e introduzir uma atribuição positiva no que diz respeito à obtenção de êxito quando surgem problemas e adversidades. Indivíduos com altos níveis de capital psicológico têm a capacidade de estruturar seu trabalho para atingir os objetivos desejados (Goertzen \& Whitaker, 2015). Levando essa conclusão em consideração, formulamos a seguinte hipótese:

H2: O capital psicológico influencia positivamente a estrutura percebida da equipe.

\section{A estrutura percebida da equipe e a aprendizagem interna em equipes}

A estrutura percebida da equipe permite que as ações dos membros da equipe sejam moldadas e pode ser aplicável a vários tipos de equipes e unidades organizacionais percebidas (Bresman \& Zellmer-Bruhn, 2013). Equipes com percepções coletivas de alto nível têm mais probabilidade de reconhecer e aproveitar oportunidades favoráveis ao processo de aprendizagem (Jansen, Kostopoulos, Mihalache, \& Papalexandris, 2016).

Para Noe, Clarke e Klein (2014), a estrutura percebida da equipe facilita o processo de aprendizagem, justamente porque promove interações entre os membros da equipe. Nesse contexto, a aprendizagem pressupõe que as atividades e esforços individuais dos membros da equipe sejam orientados para o atingimento de objetivos definidos por um processo de formalização e especialização das atividades (Bunderson \& Reagans, 2011).

0 processo de aprendizagem depende da existência de objetivos definidos e tarefas compartilhadas dentro da equipe (Mathieu, Tannenbaum, Donsbach, \& Alliger, 2014). Portanto, à medida que a estruturação do trabaIho tem se tornado cada vez mais importante na educação e no ambiente profissional, mais ênfase está sendo colocada no processo de aprendizagem e na capacidade individual de aprendizagem (Kayes et al., 2005). Por essas razões, argumentamos que a estrutura percebida da equipe contribui para o aprendizado interno. Assim, assumimos a hipótese abaixo:

H3: A estrutura percebida da equipe influencia positivamente o aprendizado interno nas equipes.

\section{O papel mediador da estrutura percebida da equipe}

Pesquisas anteriores têm dedicado pouca atenção ao papel mediador da estrutura percebida da equipe no desenvolvimento de processos de aprendizagem com foco interno (Bresman \& Zellmer-Bruhn, 2013). Da mesma forma, Bunderson e Boumgarden (2010) argumentam que a estrutura percebida da equipe é uma categoria das características de uma equipe que os pesquisadores, muitas vezes, ignoram. Para Crawford e Lepine (2013), essas teorias de processo de equipe têm se concentrado mais no conteúdo e na relevância temporal do trabalho.

Argumentamos que a estrutura percebida da equipe pode mediar a relação entre o capital psicológico e a aprendizagem interna. Refletindo sobre o papel mediador da estrutura percebida da equipe, Bunderson e Boumgarden (2010) enfatizam que ela tem implicações importantes para a aprendizagem individual com foco interno.

0 capital psicológico permite que as atitudes dos estudantes sejam influenciadas quando estes estruturam suas atividades e tarefas, o que pode criar condições favoráveis ao processo de aprendizagem interna. Como destacam Nielsen, Newman, Smyth, Hirst e Heilemann (2016), indivíduos com alto nível de PsyCap possuem a confiança (autoeficácia) necessária para lidar com as tarefas desafiadoras que enfrentam no trabalho, e acreditam que serão capazes de enfrentar esses desafios (otimismo) por meio de um redirecionamento de caminhos para facilitar objetivos (esperança) e se recuperar das adversidades (resiliência). Para Nigah, Davis e Hurrell (2012), essas habilidades são consideradas recursos psicológicos pessoais que os indivíduos podem utilizar 
para estruturar tanto suas tarefas quanto as relações que se estabelecem de acordo com as tarefas definidas, o que contribui para o processo de aprendizagem individual.

Nesse sentido, argumentamos que a estrutura percebida da equipe funciona como um mecanismo mediador, ligando o capital psicológico à aprendizagem do estudante. Embora haja poucas evidências do efeito do capital psicológico na aprendizagem interna por meio da estrutura percebida da equipe, há evidências crescentes de que o capital psicológico está ligado a uma tendência que mantém a cognição e as avaliações de um indivíduo e permite que ele lide com uma variedade de situações. Tais situações incluem um indivíduo com confiança para realizar tarefas desafiadoras, gerar soluções alternativas na adversidade, perseverar em circunstâncias difíceis, recuperar-se rapidamente dos fracassos e aprender com eles (Harms, Krasikova, \& Luthans, 2018). Essas evidências nos levam a formular a seguinte hipótese:

H4: A estrutura percebida da equipe medeia a relação entre o PsyCap e a aprendizagem interna em equipes.

A Figura 1 apresenta um modelo conceitual do papel mediador da estrutura percebida da equipe na relação entre capital psicológico e aprendizagem interna em equipes. 0 modelo conceitual também apresenta as hipóteses estudadas.

\section{Figura 1. Modelo conceitual da relação entre capital psicológico, estrutura percebida da equipe e aprendizagem interna em equipes}

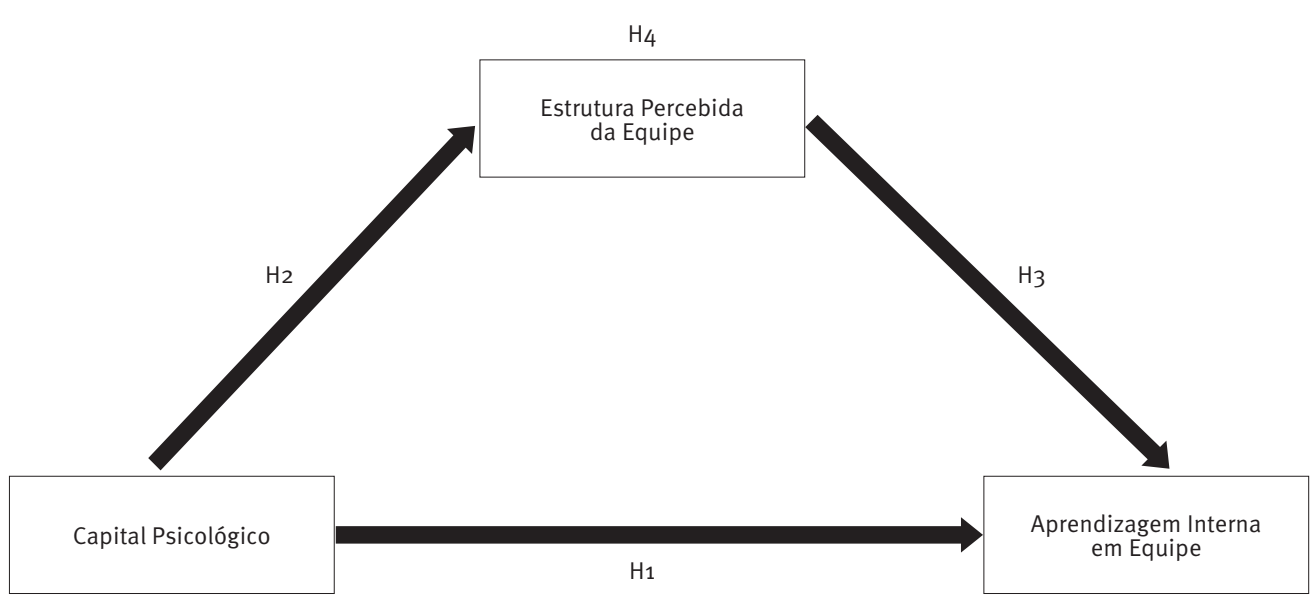

\section{MÉTODO}

\section{Participantes e procedimento}

Os participantes do estudo eram alunos de graduação de três grandes instituições de ensino superior (duas públicas e uma privada). Essas instituições de ensino superior foram selecionadas a partir de uma lista de oito instituições. Elas foram selecionadas por amostragem aleatória, conforme sugerido por Lohr (1999).

Participaram deste estudo alunos de 13 diferentes cursos de graduação (incluindo Psicologia, Pedagogia, Psicologia Clínica e Organizacional, Linguística-Português, Geografia, Fisioterapia, Contabilidade e Tributação, Biologia e História). A coleta dos dados foi autorizada pela direção de cada instituição e permitida também pelo docente de cada um dos módulos em que ocorreu a pesquisa. 
Os participantes responderam voluntariamente e pessoalmente ao questionário, durante o período de aula, utilizando papel e lápis. Responderam também a um questionário sobre seu trabalho em equipe com relação a unidades específicas do curso. Como as duas variáveis do estudo (estrutura percebida da equipe e aprendizagem interna) foram obtidas de alunos que trabalhavam em equipes, também pedimos que pensassem sobre seu trabalho individual com outros membros da equipe, e não sobre o trabalho da equipe como um todo, conforme recomendado por Lee, Kwon, Shin, Kim e Park (2018). Tem sido demonstrado, também, que, para medir um constructo em nível individual de análise sobre indivíduos que trabalhavam em equipes, eles precisam responder tomando como referência um membro da equipe (Jehn, 1995).

Durante essa fase da pesquisa, 600 questionários foram distribuídos, e 480 questionários válidos (uma taxa de aceitação de respostas de $80 \%$ ) foram recebidos de volta durante o mês de agosto de 2018 . Todos os participantes foram informados de que a participação era voluntária e que os dados coletados seriam tratados pelos pesquisadores envolvidos no estudo de modo confidencial e utilizados apenas para os fins do estudo. Todas as dúvidas dos participantes foram esclarecidas durante o processo de coleta de dados.

Dos participantes, 54\% eram homens, e sua idade média era de 24 anos (as idades variavam de 17 a 58 anos). Os cursos mais significativos foram Economia (25\%), Administração (12\%), Enfermagem (11\%) e Linguística-Inglês ( $8 \%$ ), sendo que $64 \%$ dos participantes estudavam no período da manhã, $2 \%$, à tarde e $34 \%$, no período após o trabalho. Além disso, $61 \%$ dos alunos estavam no primeiro ano, 21\%, no segundo ano, 11\%, no terceiro ano e $7 \%$, no quarto ano.

\section{Medidas}

Capital Psicológico Utilizamos a versão do questionário de 24 itens, adaptada para pesquisas acadêmicas por Luthans et al. (2012). Os 24 itens que medem o capital psicológico foram adaptados a partir de escalas publicadas já utilizadas em estudos anteriores, como os de Luthans e Luthans (2014) e Luthans, Luthans e Palmer (2016).

A escala é composta por quatro subescalas com seis itens cada, correspondendo a capacidades psicológicas positivas, que avaliam: autoeficácia (ex.: "Sinto-me confiante quando procuro uma solução para um problema de longo prazo"); esperança (ex.: "Se eu me encontrasse em uma situação difícil em um trabalho do curso, eu conseguiria pensar em muitas maneiras de sair dela"); resiliência (ex.: "Quando me deparo com um obstáculo em meus estudos, acho difícil me recuperar e seguir em frente "); otimismo (ex.: “Em termos de estudos, estou otimista com o que vai acontecer no futuro").

As respostas foram dadas em uma escala Likert de 6 pontos, de (1) "Discordo Totalmente" a (6) "Concordo Totalmente”. De acordo com Luthans et al. (2012), a escala do PsyCap de 24 itens apresentada no estudo original tem um a de Cronbach de 0,90 .

Estrutura percebida da equipe. Utilizou-se a escala desenvolvida e validada por Bunderson e Boumgarden (2010), composta por nove itens com duas subescalas, a saber, especialização (ex.: "Nossas tarefas individuais são muito claras e não nos afastamos delas") e formalização (ex.: "Seguimos um programa de trabalho bastante estruturado"). 0 a de Cronbach relatado pelos autores é de 0,75. Em um estudo posterior, Bresman e Zellmer-Bruhn (2013) validaram a escala e obtiveram um a de Cronbach de 0,73 . As respostas foram dadas em uma escala Likert de 7 pontos, de (1) "Discordo Totalmente" a (7) “Concordo Totalmente”.

Aprendizagem interna em equipes. Utilizamos a escala desenvolvida e inicialmente validada por Edmondson (1999), e posteriormente confirmada e validada por Bresman e Zellmer-Bruhn (2013), referente à aprendizagem interna e contendo sete itens. Como exemplos dos itens, temos: "Regularmente reservamos tempo para encontrar 
maneiras de melhorar os processos de trabalho do grupo" e "As pessoas da equipe frequentemente expressam suas opiniões para testar suposições sobre os assuntos em discussão". A escala de respostas utilizada é do tipo Likert de 7 pontos, de (1) “Discordo Totalmente" a (7) "Concordo Totalmente", com um a de Cronbach de 0,71.

A escala completa com 40 itens foi traduzida para o português pelo método de tradução/retroversão. A escala original e as versões traduzidas foram cuidadosamente comparadas, nessa fase, por um falante nativo de inglês, com um docente de português-inglês nos auxiliando nesse processo.

Variáveis controle. Pesquisas anteriores (por exemplo, Schneider \& Preckel, 2017) argumentaram que os resultados de aprendizagem dos alunos podem ser influenciados pelo ano em que se encontram no curso. Assim, incluímos essa variável demográfica em nossas análises. 0 ano do curso foi uma medida autodeclarada.

\section{Validade das medições}

Realizamos uma análise fatorial confirmatória para cada construto, a fim de examinar sua estrutura fatorial. A qualidade do ajuste local do modelo baseou-se nos pesos fatoriais e na confiabilidade dos itens individuais. Também examinamos a validade convergente (ou seja, a confiabilidade composta) (Fornell \& Larcker, 1981).

A análise fatorial confirmatória, realizada com o software Amos na escala de PsyCap, resultou em valores adequados. 0 modelo apresenta pesos fatoriais moderados e bons $(\lambda \geq 0,30)$ e confiabilidades individuais adequadas ( $(r 2 \geq 0,10)$. 0 modelo final tem índices de ajuste excelentes ${ }^{*}\left(c^{2}(145)=242,993, r<0,001 ; \mathrm{TLI}=0,908\right.$; CFI

= 0,922; GFI = 0,949; SRMR = 0,044; RMSEA = 0,038). 0 a de Cronbach para a dimensão de PsyCap foi de o,86. É importante enfatizar que, neste estudo, utilizamos o PsyCap como fator de segunda ordem, uma vez que vários estudos têm mostrado que o PsyCap como construto de segunda ordem possui um impacto mais forte nos resultados do que as quatro capacidades psicológicas tomadas separadamente (Alessandri, Consiglio, Luthans, \& Borgogni, 2018; Avey, Reichard, Luthans, \& Mhatre, 2011; Badran \& Youssef-Morgan, 2015).

Foram obtidos valores adequados por meio de análise fatorial confirmatória para a escala da Estrutura Percebida da Equipe. 0 modelo possui pesos fatoriais moderados e bons $(\lambda \geq 0,40)$ e confiabilidades individuais adequadas $(r 2 \geq 0,16)$. 0 modelo final tem bons índices de ajuste $c^{2}(26)=89,137, r<0,001 ; T L I=0,899$; $C F I$ $=0,927 ; \mathrm{GFI}=0,962 ; \mathrm{SRMR}=0,045 ; \mathrm{RMSEA}=0,071.0$ a de Cronbach para a estrutura da equipe foi de 0,82 .

Foram obtidos valores adequados por meio de análise fatorial confirmatória para a escala da Aprendizagem Interna. 0 modelo possui pesos fatoriais moderados e bons $(\lambda \geq 0,40)$ e confiabilidades individuais adequadas $(\mathrm{r} 2 \geq 0,16)$. $O$ modelo final tem índices de ajuste excelentes $\left(c^{2}(7)=23,797, r<0,001 ; T L I=0,950 ; C F I=0,977\right.$; $\mathrm{GFI}=0,984 ; \mathrm{SRMR}=0,034 ; \mathrm{RMSEA}=0,071) .0$ coeficiente a de Cronbach para aprendizagem interna é de 0,76.

\section{RESULTADOS}

\section{Estatísticas descritivas}

A Tabela 1 apresenta as médias, desvios-padrão, as de Cronbach (entre parênteses) e correlações de Pearson das variáveis em estudo. De acordo com a Tabela 1, as consistências internas são geralmente aceitáveis. 0 modelo da mediação da estrutura percebida da equipe na aprendizagem interna foi ajustado para 480 participantes. O modelo possui um ajuste aceitável $\left(c^{2}(450)=716,726, r<0,001 ; \mathrm{TLI}=0,90 \mathrm{CFI}=0,902 ; \mathrm{GFI}=0,915\right.$; SRMR = 0,$050 ;$ RMSEA = 0,035). 
Utilizamos um programa de análise de poder de modelagem de equações estruturais com um tamanho de efeito previsto de 0,144 em um nível de probabilidade de 0,05 e um nível de poder estatístico de 0,80, conforme sugerido por Westland (2010). Os resultados mostraram que, para se ter um tamanho amostral aceitável para o teste das hipóteses de pesquisa, este estudo precisaria de um mínimo de 379 participantes. Consequentemente, argumentamos que o tamanho da amostra do presente estudo ( 480 alunos) indica que o poder explicativo é aceitável.

Tabela 1. Médias, desvios-padrão e correlações entre as variáveis estudadas

\begin{tabular}{l|c|c|c|c|c|c|c}
\hline Variáveis estudadas & M & DP & Máximo & Mínimo & $\mathbf{1}$ & $\mathbf{2}$ & $\mathbf{3}$ \\
\hline 1. Ano do curso & 1,63 & 0,93 & 4 & 1 & - & $\mathbf{4}$ \\
\hline $\begin{array}{l}\text { 2. Capital psicológico } \\
\text { 3. Estrutura percebida }\end{array}$ & 4,75 & 0,57 & 5,90 & 2,04 & 0,031 & $(0,86)$ & \\
\hline $\begin{array}{l}\text { da equipe } \\
\text { 4. Aprendizagem } \\
\text { interna em equipes }\end{array}$ & 4,46 & 1,08 & 7,00 & 1,00 & $-0,099^{*}$ & $0,335^{* *}$ & $(0,82)$ \\
\hline
\end{tabular}

$N=480$; as de Cronbach (entre parênteses)

** A correlação é significativa no nível de 0,01 (bicaudal).

\section{Avaliação do viés do método comum}

Richardson, Simmering e Sturman (2009) apontaram que o viés do método comum pode ter implicações significativas para as relações entre as medições de diferentes construtos. Para Podsakoff, MacKenzie, Lee e Podsakoff (2003), o uso de "remédios estatísticos" pode minimizar os efeitos do viés do método comum sobre os resultados do estudo. Uma vez que os dados deste estudo foram obtidos da mesma fonte, avaliamos o efeito dessa variação por meio da reestimação do modelo de medição, adicionando um fator de método comum latente (teste de fator único de Harman).

0 ajuste do modelo sem o fator de método comum produziu os seguintes índices de ajuste: $\left(c^{2}(422)=\right.$ 663,455, $\mathrm{r}<0,001 ; \mathrm{TLI}=0,901 ; \mathrm{CFI}=0,911 ; \mathrm{GFI}=0,918 ; \mathrm{SRMR}=0,049 ; \mathrm{RMSEA}=0,035)$. Embora a inclusão do fator de método comum tenha melhorado apenas ligeiramente o ajuste do modelo em certos índices, os resultados são os seguintes: $\left(c^{2}(421)=666,986, \mathrm{r}<0,001 ; \mathrm{TLI}=0,899 ; \mathrm{CFI}=0,909 ; \mathrm{GFI}=0,919 ; \mathrm{SRMR}=0,039\right.$; RMSEA = 0,035).

No entanto, a proporção da variância total explicada por esse fator de método foi de $15 \%$, abaixo dos $25 \%$ sugeridos por Williams, Hartman e Cavazotte (2010). Argumentamos, portanto, que o viés de mesma fonte não pode ser considerado uma ameaça aos resultados deste estudo.

\section{Testes das hipóteses}

Com relação aos testes das hipóteses, adotamos uma abordagem bootstrap com intervalo de confiança de $90 \%$ sobre os efeitos indiretos padronizados. 0 modelo ajustado explica $55 \%$ da variabilidade da Aprendizagem Interna. Os resultados mostram que o capital psicológico tem uma influência positiva e estatisticamente significativa sobre a aprendizagem interna $(\beta=0,113 ;=0,006)$. A hipótese $\mathrm{H}_{1}$ está, portanto, apoiada. 0 PsyCap teve uma influência significativa e positiva sobre a estrutura percebida da equipe $(\beta=0,45 ;<0,001)$, apoiando $\mathrm{H} 2$. A estrutura percebida da equipe está associada de maneira positiva e significativa à aprendizagem interna $(\beta=$ 
0,$73 ; \mathrm{r}(0,001)$, o que apoia $\mathrm{H}_{3}$. Os resultados mostram que a estrutura percebida da equipe medeia totalmente a relação entre o capital psicológico e a aprendizagem interna (efeito indireto = 0,33; IC 90\%, limites ] 0,22, 0,46 D, apoiando, assim, a hipótese $\mathrm{H}_{4}$.

\section{Figura 2. Modelo final}

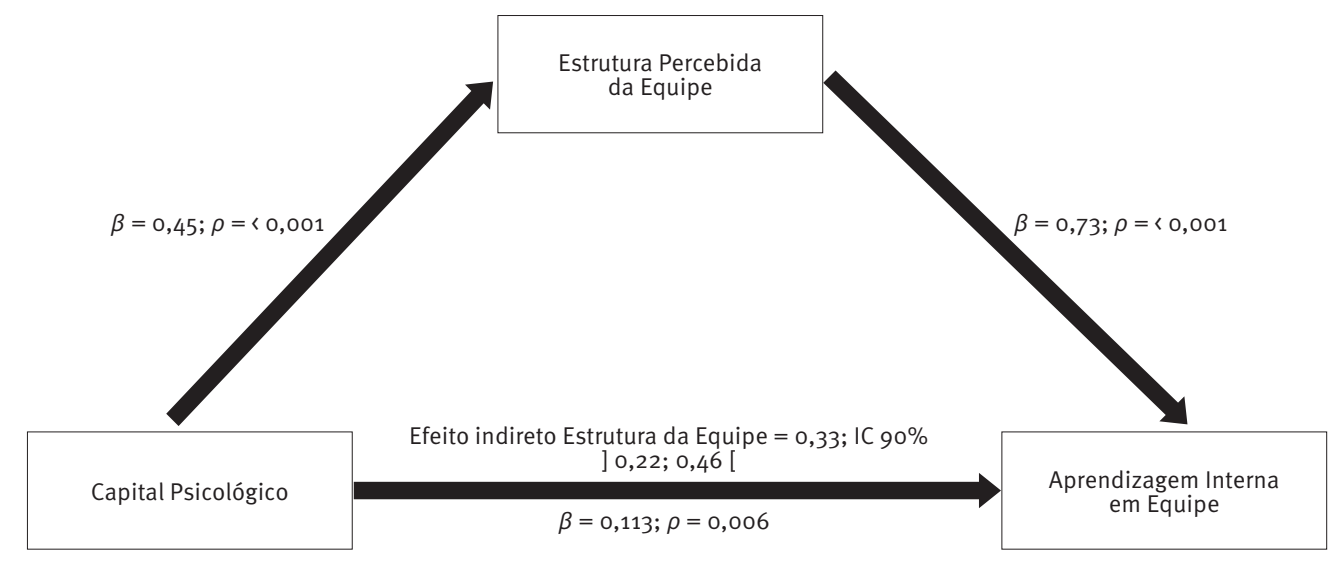

\section{DISCUSSÃO}

O objetivo deste estudo foi analisar o papel mediador da estrutura percebida da equipe na relação entre capital psicológico e aprendizagem interna. Os resultados confirmaram a existência de uma relação positiva entre capital psicológico e aprendizagem interna. Isso destaca a importância que as capacidades psicológicas positivas possuem no sentido de favorecer uma maior força e um estado mais alerta dos indivíduos, contribuindo, assim, para a aprendizagem interna (Chen \& Lim, 2012).

Indivíduos com essas capacidades psicológicas realizam esforços que, quando bem executados, levam a certos resultados bem-sucedidos (Stajkovic \& Luthans, 1998). Essa relação entre capital psicológico e aprendizagem interna parece ocorrer devido à forte ligação entre capital psicológico e resultados acadêmicos bem-sucedidos (Luthans, Luthans, \& Chaffin, 2018). As atividades de aprendizagem também requerem esforços concretos e substanciais, como a identificação de problemas e a busca de informações para resolvê-los (Huang \& Luthans, 2014).

A relação entre capital psicológico e estrutura percebida da equipe também foi confirmada. Como relataram Luthans et al. (2018), indivíduos com altos níveis de capital psicológico lidam melhor com erros, fracassos e revezes, e geralmente não permitem que circunstâncias difíceis os impeçam de atingir altos níveis de desempenho. Esses fatores podem ser suficientes para estimular os indivíduos a buscarem outros caminhos relevantes para uma definição clara de seus objetivos e procedimentos. 0 capital psicológico também é importante para revelar os fatores psicológicos individuais relacionados ao processo de estruturação (Daspit et al., 2015).

Um estudo longitudinal (em um intervalo de quatro semanas) com 391 estudantes universitários holandeses mostrou que recursos pessoais como autoeficácia, esperança, otimismo e resiliência são realmente importantes para prever um maior envolvimento dos alunos na obtenção de melhores resultados (Ouweneela, Blanca, \& Schaufeli, 2011). 
A terceira hipótese sobre a relação positiva entre a estrutura percebida da equipe e a aprendizagem interna também foi confirmada. Esse resultado parece confirmar o que foi relatado por Bunderson e Boumgarden (2010), isto é, que a identificação de papéis entre os membros do grupo facilita o compartilhamento de informações e possibilita a realização de tarefas que podem impulsionar o processo de aprendizagem. Estrutura é fundamental para o processo de aprendizagem, pois a aprendizagem requer algum grau de confiança, bem como potencial para atingir o desempenho desejado. Isso, por sua vez, requer uma definição clara de objetivos e procedimentos (Yoon \& Kayes, 2016).

Por fim, foi confirmado o papel mediador da estrutura percebida da equipe na relação entre capital psicológico e aprendizagem interna. Esse resultado é consistente com pesquisas anteriores (por exemplo, Bunderson \& Boumgarden, 2010), que argumentam que a estrutura percebida pode promover a aprendizagem e criar um ambiente seguro dentro da equipe, facilitando, assim, o compartilhamento de informações. A estrutura percebida das atividades também tende a propiciar a experimentação e a busca de novos insights, o que pode ter um impacto positivo no processo de aprendizagem (Bunderson \& Boumgarden, 2010).

\section{CONCLUSÕES}

Estudos realizados ao longo dos anos têm mostrado que o capital psicológico está relacionado a vários resultados importantes, tanto dentro como fora do ambiente de trabalho (por exemplo, Dawkins et al., 2013; Krasikova, Lester, \& Harms, 2015; Luthans, Youssef, \& Rawski, 2011; Luthans \& Youssef-Morgan, 2017; Newman et al., 2014). 0 objetivo deste estudo foi analisar o papel mediador da estrutura percebida da equipe na relação entre capital psicológico e aprendizagem interna. 0 estudo forneceu evidências empíricas da influência do capital psicológico sobre o processo de aprendizagem interna por meio da estrutura percebida da equipe. Portanto, argumentamos que este estudo é importante não apenas porque pode ser aplicado em ambientes acadêmicos para potencializar o processo de aprendizagem do aluno, mas também porque levanta novas questões teóricas e desafios para estudos futuros.

\section{Implicações teóricas e práticas}

0 estudo contribui para a literatura de diferentes maneiras. Fornecemos evidências de que a relação entre capital psicológico e aprendizagem interna é mediada pela estrutura percebida da equipe. Isso confirma que uma das características distintivas do capital psicológico, e que tem uma importante contribuição prática a dar, é sua abertura a mudanças e ao desenvolvimento (Choi \& Lee, 2014; Han, Brooks, Kakabadse, Peng, \& Zhu, 2012; Nwanzu \& Babalola, 2019).

Ele também fornece evidências empíricas adicionais à ideia de que as capacidades psicológicas, como autoeficácia, esperança, otimismo e resiliência, podem ser utilizadas para aprimorar o processo de aprendizagem interna da equipe. Esses resultados são importantes porque ressaltam a necessidade de intervenções formativas voltadas ao desenvolvimento das capacidades psicológicas (Rebelo, Dimas, Lourenço, \& Palácio, 2018). Estudos realizados por Luthans e Youssef-Morgan (2017) trazem insights e diretrizes práticas para o desenvolvimento do PsyCap. 


\section{Limitações e futuros direcionamentos}

Este estudo tem suas limitações. Uma delas diz respeito ao potencial problema do viés do método comum, uma vez que utilizamos a mesma fonte para a coleta de dados. Ao abordar essa questão nos resultados do estudo, utilizamos “remédios estatísticos" como o método do fator latente. Os resultados mostram que o viés do método comum não pode ser considerado uma ameaça aos resultados deste estudo. No entanto, pesquisas adicionais podem atenuar o potencial problema do viés do método comum por meio da coleta de dados de várias fontes (por exemplo, pela obtenção de medidas do capital psicológico de alunos, docentes e gestores de instituições de ensino superior).

Por outro lado, o questionário sobre o PsyCap utilizado neste estudo é tradicionalmente aplicado em ambientes organizacionais. Embora tenhamos utilizado a versão do questionário adaptada por Luthans (2012) para pesquisas acadêmicas, encorajamos futuros pesquisadores a aprofundarem o estudo da aplicabilidade do instrumento em outros contextos, fora do ambiente de trabalho.

\section{NOTAS DOS AUTORES}

*Parâmetros de aceitabilidade da Modelagem de Equações Estruturais (Hair, Anderson, Tatham, \& Black, 1995):

- Qui-quadrado (x2): valor- $p \leq 0,05$.

- Indice de bondade do ajuste (GFI): $\geq 0,90$.

- Indice de ajuste comparativo (CFI): $\geq 0,90$.

- Índice de Tucker-Lewis (TLI): $\geq 0,90$.

- Raiz do erro quadrático médio de aproximação (RMSEA): $\leq 0,08$

- Raiz do resíduo quadrático médio padronizado (SRMR): $\leq 0,08$

\section{AGRADECIMENTOS}

1. Este trabalho foi apoiado por fundos nacionais portugueses através da Fundação para a Ciência e a Tecnologia (FCT), no âmbito do projeto UIDP / 00713/2021.

2. Agradecemos ao Editor-Chefe e aos dois pareceristas anônimos por sua significativa contribuição para a melhoria deste estudo.

\section{REFERÊNCIAS}

Alessandri, G., Consiglio, C., Luthans, F., \& Borgogni, L. (2018). Testing a dynamic model of the impact of psychological capital on work engagement and job performance. Career Development International, 23(1), 33-47. doi:10.1108/CDI-112016-0210

Argote, L., \& Miron-Spektor, E. (2011). Organizational learning: From experience to knowledge. Organization Science, 22(5), 1123-1137. doi:10.1287/orsc.1100.0621

Avey, J. B. (2014). The left side of psychological capital: New evidence on the antecedents of PsyCap. Journal of Leadership and Organizational Studies, 21(2), 141-149. doi:10.1177/1548051813515516

Avey, J. B., Reichard, R. J., Luthans, F., \& Mhatre, K. H. (2011). Meta-analysis of the impact of positive psychological capital on employee attitudes, behaviors, and performance. Human Resource Development Quarterly, 22(2), 127-152. doi:10.1002/ hrdq. 20070
Badran, M. A., \& Youssef-Morgan, C. M. (2015). Psychological capital and job satisfaction in Egypt. Journal of Managerial Psychology, 30(3), 354-379. doi:10.1108/JMP-06-2013-0176

Bandura, A. (1982). Self-efficacy mechanism in human agency. American Psychologist, 37(2), 122-147. doi:10.1037/0003066X.37.2.122

Bresman, H. (2010). External learning activities and team performance: A multimethod field study. Organization Science, 21(1), 81-96. doi:10.1287/orsc.1080.0413

Bresman, H., \& Zellmer-Bruhn, M. (2013). The structural context of team learning: Effects of organizational and team structure on internal and external learning. Organization Science, 24(4), 1120-1139. doi:10.1287/orsc.1120.0783

Bunderson, J. S., \& Boumgarden, P. (2010). Structure and learning in self-managed teams: Why "bureaucratic" teams can be better learners. Organization Science, 21(3), 609-624. doi:10.1287/orsc.1090.0483 
Bunderson, J. S., \& Reagans, R. E. (2011). Power, status, and learning in organizations. Organization Science, 22(5), 1182 1194. doi:10.1287/orsc.1100.0590

Chen, D. J., \& Lim, V. K. (2012). Strength in adversity: The influence of psychological capital on job search. Journal of Organizational Behavior, 33(6), 811-839. doi:10.1002/job.1814

Choi, Y., \& Lee, D. (2014). Psychological capital, Big Five traits, and employee outcomes. Journal of Managerial Psychology, 29(2), 122-140. doi:10.1108/JMP-06-2012-0193

Crawford, E. R., \& Lepine, J. A. (2013). A configural theory of team processes: Accounting for the structure of taskwork and teamwork. Academy of Management Review, 38(1), 32-48. doi:10.5465/amr.2011.0206

Daspit, J. J., Mims, T. C., \& Zavattaro, S. M. (2015). The role of positive psychological states in online learning: Integrating psychological capital into the community of inquiry framework. Journal of Management Education, 39(5), 626 649. doi:10.1177/1052562914564980

Datu, J. A., \& Valdez, J. P. (2019). Psychological capital is associated with higher levels of life satisfaction and school belongingness. School Psychology International, 1(1), 1-16. doi:10.1177/0143034319838011

Dawkins, S., Martin, A., Scott, J., \& Sanderson, K. (2013). Building on the positives: A psychometric review and critical analysis of the construct of psychological capital. Journal of Occupational and Organizational Psychology, 86(3), 348-370. doi:10.1111/joop.12007

Edmondson, A. (1999). Psychological safety and learning behavior in work teams. Administrative Science Quarterly, 44(2), 350-383. doi:10.2307/2666999

Fornell, C., \& Larcker, D. F. (1981). Evaluating structural equation models with unobservable variables and measurement error. Journal of Marketing Research, 18(1), 39-50. doi:10.2307/3151312

Goertzen, B. J., \& Whitaker, B. L. (2015). Development of psychological capital in an academic-based leadership education program. Journal of Management Development, 34 (7), 773-786. doi:10.1108/jmd-07-2013-0100

Hair, J., Anderson, R., Tatham, R. L., \& Black, W. C. (1995). Multivariate data analysis. Englewood Cliffs, NJ: Prentice-Hall.

Han, Y., Brooks, I., Kakabadse, N. K., Peng, Z., \& Zhu, Y. (2012). A grounded investigation of Chinese employees' psychological capital. Journal of Managerial Psychology, 27(7), 669-695. doi:10.1108/02683941211259511

Harms, P. D., Krasikova, D. V., \& Luthans, F. (2018). Not me, but reflects me: Validating a simple implicit measure of psychological capital. Journal of Personality Assessment, 100 (5), 1-12. doi:10.1080/00223891.2018.1480489

Harms, P. D., \& Luthans, F. (2012). Measuring implicit psychological constructs in organizational behavior: An example using psychological capital. Journal of Organizational Behavior, 33(4), 589-594. doi:10.1002/job.1785

Heled, E., Somech, A., \& Waters, L. (2015). Psychological capital as a team phenomenon: Mediating the relationship between learning climate and outcomes at the individual and team levels. The Journal of Positive Psychology, 11(3), 303-314. doi :10.1080/17439760.2015.1058971

Herrmann, K. J. (2013). The impact of cooperative learning on student engagement: Results from an intervention. Active Learning in Higher Education, 14(3), 175-187. doi:10.1177/1469787413498035
Huang, L., \& Luthans, F. (2014). Toward better understanding of the learning goal orientationn-creativity relationship: The role of positive psychological capital. Applied Psychology, 64(2), 444-472. doi:10.1111/apps.12028

Jansen, J. J., Kostopoulos, K. C., Mihalache, O. R., \& Papalexandris, A. (2016). A socio-psychological perspective on team ambidexterity: The contingency role of supportive leadership behaviours. Journal of Management Studies, 53(6), 939-965. doi:10.1111/joms.12183

Jehn, K. A. (1995). A multimethod examination of the benefits and detriments of intragroup conflict. Administrative Science Quarterly, 40(2), 256-282. doi:10.2307/2393638

Jiang, Y., Jackson, S. E., \& Colakoglu, S. (2015). An empirica examination of personal learning within the context of teams. Journal of Organizational Behavior, 37(5), 654-672. doi:10.1002/job.2058

Kayes, A. B., Kayes, D. C., \& Kolb, D. A. (2005). Experiential learning in teams. Simulation \& Gaming, 36(3), 330-354. doi:10.1177/1046878105279012

King, D. D., Newman, A., \& Luthans, F. (2015). Not if, but when we need resilience in the workplace. Journal of Organizational Behavior, 37(5), 782-786. doi:10.1002/job.2063

Kostopoulos, K. C., Spanos, Y. E., \& Prastacos, G. P. (2013). Structure and function of team learning emergence: A multilevel empirical validation. Journal of Management, 39(6), 1430-1461. doi:10.1177/0149206311419366

Krasikova, D. V., Lester, P. B., \& Harms, P. D. (2015). Effects of psychological capital on mental health and substance abuse. Journal of Leadership \& Organizational Studies, 22(3), 280291. doi:10.1177/1548051815585853

Lee, S., Kwon, S., Shin, S. J., Kim, M., \& Park, I.-J. (2018) How team-level and individual-level conflict influences team commitment: A multilevel investigation. Frontiers in Psychology, 8(1), 1-13. doi:10.3389/fpsyg.2017.02365

Lohr, S. (1999). Sampling: Design and analysis. Pacific Grove, CA: Duxbury Press.

Luthans, B. C., \& Luthans, K. W. (2014). Building the leaders of tomorrow: The development of academic psychological capital. Journal of Leadership \& Organizational Studies, 2o(10), 191-199. doi:10.1177/1548051813517003

Luthans, B. C., Luthans, K. W., \& Jensen, S. M. (2012). The impact of business school students' psychological capital on academic performance. Journal of Education for Business, 87(5), 253-259. doi:10.1080/08832323.2011.609844

Luthans, F. (2012). The need for and meaning of positive organizational behavior. Journal of Organizational Behavior 23(6), 695-706. doi:10.1108/CDI-11-2016-0210

Luthans, F., Avey, J. B., Avolio, B. J., Norman, S. M., \& Combs, G. M. (2006). Psychological capital development: Toward a micro-intervention. Journal of Organizational Behaviour, 27(3), 387-393. doi:10.1002/job.373

Luthans, F., Avey, J. B., Avolio, B. J., \& Peterson, S. J. (2010). The development and resulting performance impact of positive psychological capital. Human Resource Development Quarterly, 21(1), 41-67. doi:10.1002/hrdq.20034

Luthans, F., Youssef, C. M., \& Rawski, S. L. (2011). A tale of two paradigms: The impact of psychological capital and reinforcing feedback on problem solving and innovation. Journal of Organizational Behavior Management, 31(4), 333350. doi:10.1080/01608061.2011.619421 
Luthans, F., \& Youssef-Morgan, C. M. (2017). Psychological capital: An evidence-based positive approach. Annual Review of Organizational Psychology and Organizational Behavior, 4(1), 339-366. doi:10.1146/annurev-orgpsych-032516-113324

Luthans, K. W., Luthans, B. C., \& Chaffin, D. (2018). Refining grit in academic performance: The mediational role of psychological capital. Journal of Management Education, 43(1), 1-27. doi:10.1177/1052562918804282

Luthans, K. W., Luthans, B. C., \& Palmer, N. F. (2016). A positive approach to management education: The relationship between academic PsyCap an student engagement. Journal of Management Development, 35(9), 1-22. doi:10.1108/JMD06-2015-0091

Mathieu, J. E., Tannenbaum, S. I., Donsbach, J. S., \& Alliger, G. M. (2014). A review and integration of team composition models: Moving toward a dynamic and temporal framework. Journal of Management, 40(1), 130-160. doi:10.1177/0149206313503014

Newman, A., Ucbasaran, D., Zhu, F., \& Hirst, G. (2014). Psychological capital: A review and synthesis. Journal of Organizational Behavior, 35(1), 120-138. doi:10.1002/job.1916

Nielsen, I., Newman, A., Smyth, R., Hirst, G., \& Heilemann, B. (2016). The influence of instructor support, family support and psychological capital on the well-being of postgraduate students: A moderated mediation model. Studies in Higher Education, 42(11), 2099-2115. doi:10.1080/03075079.2015.1 135116

Nigah, N., Davis, A. J., \& Hurrell, S. A. (2012). The impact of buddying on psychological capital, work engagement and turnover intentions: An empirical study of socialization in the professional services sector. Thunderbird International Business Review, 54(6), 891-905. doi:10.1002/tie.21510

Noe, R. A., Clarke, A. D., \& Klein, H. J. (2014). Learninginthe twentyfirst century workplace. Annual Review of Psychology, 9(1), 245275. doi:10.1146/annurevannurevorgpsych-031413-091321

Nwanzu, C. L., \& Babalola, S. S. (2019). Examining psychological capital of optimism, self-efficacy and self-monitoring as predictors of attitude towards organizational change. International Journal of Engineering Business Management, 11(1), 1-12. doi:10.1177/1847979019827149

Ortega-Maldonado, A., \& Salanova, M. (2017). Psychological capital and performance among undergraduate students: The role of meaning-focused coping and satisfaction. Teaching in Higher Education, 23(3), 390-402. doi:10.1080/13562517.201 7.1391199

Ouweneela, E., Blanca, P. M., \& Schaufeli, W. B. (2011). Flourishing students: A longitudinal study on positive emotions, personal resources, and study engagement. Journal of Positive Psychology, 6(2), 142-153. doi:10.1080/17439760.2011.558847

Peterson, S. J., Luthans, F., Avolio, B. J., Walumbwa, F. O., \& Zhang, Z. (2011). Psychological capital and employee performance: A latent growth modeling approach. Personnel Psychology, 64(2), 427-450. doi: 10.1111/j.1744-6570.2011.01215.x
Podsakoff, P. M., MacKenzie, S. B., Lee, J.-Y., \& Podsakoff, N. P. (2003). Common method biases in behavioral research: A critical review of the literature and recommended remedies. Journal of Applied Psychology, 88(5), 879-903. doi:10.1037/0021-9010.88.5.879

Rebelo, T., Dimas, I. D., Lourenço, P. R., \& Palácio, Â. (2018). Generating team PsyCap through transformational leadership. Team Performance Management: An International Journal, 24(7/8), 363-379. doi:10.1108/tpm-09-2017-0056

Richardson, H. A., Simmering, M. J., \& Sturman, M. C. (2009). A tale of three perspectives. Organizational Research Methods, 12(4), 762-80o. doi:10.1177/1094428109332834

Salas, E., Shuffler, M. L., Thayer, A. L., Bedwell, W., \& Lazzara, E. H. (2014). Understanding and improving teamwork in organizations: A scientifically based practical guide. Human Resource Management, 54(4), 599-622. doi:10.1002/ hrm. 21628

Schaubroeck, J., Carmeli, A., Bhatia, S., \& Paz, E. (2016). Enabling team learning when members are prone to contentious communication: The role of team leader coaching. Human Relations, 69(8), 1709-1727. doi:10.1177/0018726715622673

Siu, O. L., Bakker, A. B., \& Jiang, X. (2014). Psychological capital among university students: Relationships with study engagement and intrinsic motivation. Journal of Happiness Studies, 15(4), 979-994. doi:10.1007/s10902-013-9459-2

Schneider, M., \& Preckel, F. (2017). Variables associated with achievement in higher education: A systematic review of meta-analyses. Psychological Bulletin, 143(6), 565-600. doi:10.1037/bulooooog8

Song, J. H., Lim, D. H., Kang, I. G., \& Kim, W. (2014). Team performance in learning organizations: Mediating effect of employee engagement. Learning Organization, 21(5), 290309. doi:10.1108/TLO-07-2012-0049

Stajkovic, A. D., \& Luthans, F. (1998). Self-efficacy and workrelated performance: A meta-analysis. Psychological Bulletin, 124(2), 240-261. doi:10.1037/0033-2909.124.2.240

Walumbwa, F. O., Luthans, F., Avey, J. B., \& Oke, A. (2011). Authentically leading groups: The mediating role of collective psychological capital and trust. Journal of Organizational Behavior, 32(1), 4-24. doi:10.1002/job.653

Westland, C. J. (2010). Lower bounds on sample size in structural equation modeling. Electronic Commerce Research and Applications, 9(6), 476-487. doi:10.1016/j.elerap.2010.07.003

Williams, L. J., Hartman, N., \& Cavazotte, F. (2010). Method Variance and Marker Variables: A Review and Comprehensive CFA Marker Technique. Organizational Research Methods, 13(3), 477-514. doi:10.1177/1094428110366036

Yoon, J., \& Kayes, C. (2016). Employees' self-efficacy and perception of individual learning in teams: The cross-level moderat ing role of team-learning behavior. Journal of Organizational Behavior, 37(7), 1044-1060. doi:10.1002/job.2092

\section{CONTRIBUIÇÕES DOS AUTORES}

Os autores declaram que participaram de todos os estágios do desenvolvimento do manuscrito. Rosa Lutete Geremias idealizou, elaborou, executou o processo de coleta de dados e escreveu o artigo. Miguel Pereira Lopes revisou a seção da análise e discussão e corrigiu todo o manuscrito. André Escórcio Soares analisou e verificou os dados deste artigo. Todos os autores contribuíram com o artigo e aprovaram a versão submetida. 